\title{
Approximation of functions of several variables by multidimensional $S$-fractions with independent variables
}

\author{
Dmytryshyn R.I., Sharyn S.V.
}

\begin{abstract}
The paper deals with the problem of approximation of functions of several variables by branched continued fractions. We study the correspondence between formal multiple power series and the so-called "multidimensional S-fraction with independent variables". As a result, the necessary and sufficient conditions for the expansion of the formal multiple power series into the corresponding multidimensional $S$-fraction with independent variables have been established. Several numerical experiments show the efficiency, power and feasibility of using the branched continued fractions in order to numerically approximate certain functions of several variables from their formal multiple power series.

Key words and phrases: branched continued fraction, continued fraction, multiple power series, algorithm, correspondence.
\end{abstract}

Vasyl Stefanyk Precarpathian National University, 57 Shevchenko Str., 76018, Ivano-Frankivsk, Ukraine

E-mail: dmytryshynr@hotmail.com (Dmytryshyn R.I.), serhii.sharyn@pnu.edu . ua (Sharyn S.V.)

\section{Introduction}

Representation of functions of several variables by means of branched continued fractions has been an interesting matter of study during the 70 years and has given rise to other important related topics like multivariate Padé approximants, systems of linear algebraic equations, quadratic form, system of partial differential equations and so on (see, e.g., $[1,13,14,25,30,39]$ ).

Branched continued fractions, being a multidimensional generalization of continued fractions, in comparison with multiple power series under certain conditions have wider convergence domain and endowed with the property of numerical stability. This makes them an effective tool for approximating the functions of several variables. In the works $[1,4,10,16,19$, $21-23,26,29,32-34,38]$ the authors have considered different representations of the functions of several variables by means of branched continued fractions.

Construction of the rational approximations of a function of several variables is based on the concept of correspondence between the approximants of a branched continued fraction and a formal multiple power series, which represents this function (see, e.g., [1,19]). In [11], D.I. Bodnar introduced branched continued fractions, which are the analogues of multiple power series by their structure. The correspondence properties of a branched continued fraction with polynomial elements are closely connected to the degree and form of these polynomials. Some of the most important types are given in $[10,12,17,18,20]$.

Since the multidimensional $S$-fraction with independent variables is a special case of a multidimensional regular $C$-fraction with independent variables, all theorems of $[19,24]$ may 
be applied to multidimensional $S$-fractions with independent variables, but more we can say in this paper. We study here the correspondence between formal multiple power series and multidimensional $S$-fraction with independent variables. And we give some examples of representing the functions of several variables and the mathematical constants by these branched continued fractions.

\section{Multidimensional $S$-fractions with independent variables}

\subsection{Definitions and preliminaries}

Let $N$ be a fixed natural number. The following notations will be used: $\mathbb{Z}_{\geq 0}$ denotes the non-negative integers, $\mathbb{C}$ denotes the complex numbers, $\mathbb{Z}_{\geq 0}^{N}=\mathbb{Z}_{\geq 0} \times \mathbb{Z}_{\geq 0} \times \ldots \times \mathbb{Z}_{\geq 0}$ denotes the Cartesian product of $N$ copies of $\mathbb{Z}_{\geq 0}, \mathbb{C}^{N}=\mathbb{C} \times \mathbb{C} \times \ldots \times \mathbb{C}$ denotes the Cartesian product of $N$ copies of $\mathbb{C}, \mathbf{k}=\left(k_{1}, k_{2}, \ldots, k_{N}\right)$ denotes an element of $\mathbb{Z}_{>0}^{N}, \mathbf{z}=\left(z_{1}, z_{2}, \ldots, z_{N}\right)$ is an element of $\mathbb{C}^{N}$, and for $\mathbf{k} \in \mathbb{Z}_{\geq 0}^{N}$ and $\mathbf{z} \in \mathbb{C}^{N}$

$$
\mathbf{k} !=k_{1} ! k_{2} ! \ldots k_{N} !, \quad|\mathbf{k}|=k_{1}+k_{2}+\ldots+k_{N}, \quad \mathbf{z}^{\mathbf{k}}=z_{1}^{k_{1}} z_{2}^{k_{2}} \ldots z_{N}^{k_{N}}
$$

Next, let $e_{0}=(0,0, \ldots, 0), e_{k}=\left(\delta_{k, 1}, \delta_{k, 2}, \ldots, \delta_{k, N}\right)$ be a multiindex, where $1 \leq k \leq N, \delta_{i, j}$ is the Kronecker delta. Let us introduce the following sets of multiindices for $k \geq 1$

$$
\begin{aligned}
& \mathcal{I}_{k}=\left\{i(k): i(k)=\left(i_{1}, i_{2}, \ldots, i_{k}\right), 1 \leq i_{p} \leq i_{p-1}, 1 \leq p \leq k, i_{0}=N\right\}, \\
& \mathcal{E}_{k}=\left\{e_{i(k)}: e_{i(k)}=e_{i_{1}, i_{2}, \ldots, i_{k}}=e_{i_{1}}+e_{i_{2}}+\ldots+e_{i_{k}}, i(k) \in \mathcal{I}_{k}\right\} .
\end{aligned}
$$

A branched continued fraction of the form

$$
\sum_{i_{1}=1}^{N} \frac{a_{e_{i(1)}} z_{i_{1}}}{1}+\sum_{i_{2}=1}^{i_{1}} \frac{a_{e_{i(2)}} z_{i_{2}}}{1}+\sum_{i_{3}=1}^{i_{2}} \frac{a_{e_{i(3)}} z_{i_{3}}}{1}+\cdots
$$

where $a_{e_{i(k)}}>0$ for $e_{i(k)} \in \mathcal{E}_{k}$ and $k \geq 1$, is called a multidimensional $S$-fraction with independent variables.

Let $Q_{i(k)}^{(n)}(\mathbf{z})$ denotes the "tails" of $(1)$, that is $Q_{i(n)}^{(n)}(\mathbf{z}) \equiv 1, i(n) \in \mathcal{I}_{n}, n \geq 1$, and

$$
Q_{i(k)}^{(n)}(\mathbf{z})=1+\sum_{i_{k+1}=1}^{i_{k}} \frac{a_{e_{i(k+1)}} z_{i_{k+1}}}{1}+\sum_{i_{k+2}=1}^{i_{k+1}} \frac{a_{e_{i(k+2)}} z_{i_{k+2}}}{1}+\cdots+\sum_{i_{n}=1}^{i_{n-1}} \frac{a_{e_{i(n)}} z_{i_{n}}}{1},
$$

where $i(k) \in \mathcal{I}_{k}, 1 \leq k \leq n-1, n \geq 2$. If $f_{n}(\mathbf{z})$ denotes the $n$th approximant of multidimensional $S$-fraction with independent variables (1), then

$$
f_{n}(\mathbf{z})=\sum_{i_{1}=1}^{N} \frac{a_{e_{i(1)}} z_{i_{1}}}{Q_{i(1)}^{(n)}(\mathbf{z})} \quad \text { for } \quad n \geq 1
$$

If $Q_{i(k)}^{(n)}(\mathbf{z}) \neq 0$ for all $i(k) \in \mathcal{I}, 1 \leq k \leq n, n \geq 1$, and all $\mathbf{z}$ in the certain set $D, D \subset \mathbb{C}^{N}$, then for each $n \geq 1$ and $m>n$ the following formula is valid (see [5])

$$
f_{m}(\mathbf{z})-f_{n}(\mathbf{z})=(-1)^{n} \sum_{i_{1}=1}^{N} \sum_{i_{2}=1}^{i_{1}} \ldots \sum_{i_{n+1}=1}^{i_{n}} \frac{\prod_{r=1}^{n+1} a_{e_{i(r)}} z_{i_{r}}}{\prod_{r=1}^{n+1} Q_{i(r)}^{(m)}(\mathbf{z}) \prod_{r=1}^{n} Q_{i(r)}^{(n)}(\mathbf{z})} .
$$


A series of the form

$$
L(\mathbf{z})=\sum_{|\mathbf{k}| \geq 0} c_{\mathbf{k}} \mathbf{z}^{\mathbf{k}}
$$

where $c_{\mathbf{k}} \in \mathbb{C}$ for $|\mathbf{k}| \geq 0$, is called a formal multiple power series at $\mathbf{z}=\mathbf{0}$. A set of formal multiple power series at $\mathbf{z}=\mathbf{0}$ denoted by $\mathbb{L}$.

Let $R(\mathbf{z})$ be a function holomorphic in a neighbourhood of the origin $(\mathbf{z}=\mathbf{0})$. Let the mapping $\Lambda: R(\mathbf{z}) \rightarrow \Lambda(R)$ associate with $R(\mathbf{z})$ its Taylor expansion in a neighbourhood of the origin. A sequence $\left\{R_{n}(\mathbf{z})\right\}$ of functions holomorphic at the origin is said to correspond at $\mathbf{z}=\mathbf{0}$ to a formal multiple power series $L(\mathbf{z})$ if

$$
\lim _{n \rightarrow \infty} \lambda\left(L-\Lambda\left(R_{n}\right)\right)=\infty,
$$

where $\lambda$ is the function defined as follows: $\lambda: \mathbb{L} \rightarrow \mathbb{Z}_{\geq 0} \cup\{\infty\}$; if $L(\mathbf{z}) \equiv 0$ then $\lambda(L)=\infty$; if $L(\mathbf{z}) \not \equiv 0$ then $\lambda(L)=m$, where $m$ is the smallest degree of homogeneous terms for which $c_{\mathbf{k}} \neq 0$, that is $m=|\mathbf{k}|$.

If $\left\{R_{n}(\mathbf{z})\right\}$ corresponds at $\mathbf{z}=\mathbf{0}$ to a formal multiple power series $L(\mathbf{z})$, then the order of correspondence of $R_{n}(\mathbf{z})$ is defined to be

$$
v_{n}=\lambda\left(L-\Lambda\left(R_{n}\right)\right) .
$$

By the definition of $\lambda$, the series $L(\mathbf{z})$ and $\Lambda\left(R_{n}\right)$ agree for all homogeneous terms up to and including degree $\left(v_{n}-1\right)$.

A branched continued fraction of the form (1) is said to correspond at $\mathbf{z}=\mathbf{0}$ to a formal multiple power series $L(\mathbf{z})$ if its sequence of approximants $\left\{f_{n}(\mathbf{z})\right\}$ corresponds to $L(\mathbf{z})$ at $\mathbf{z}=\mathbf{0}$.

\subsection{Correspondence}

Based on the idea of proving Theorem 1 from [23], we prove the following assertion.

Theorem 1. Every multidimensional S-fraction with independent variables (1) with sequences of approximants $\left\{f_{n}(\mathbf{z})\right\}$ corresponds at the $\mathbf{z}=\mathbf{0}$ to a uniquely determined formal multiple power series

$$
L(\mathbf{z})=\sum_{|\mathbf{k}| \geq 1}(-1)^{\mathbf{k}} c_{\mathbf{k}}(-\mathbf{z})^{\mathbf{k}}=\sum_{|\mathbf{k}| \geq 1} \gamma_{\mathbf{k}}(-\mathbf{z})^{\mathbf{k}},
$$

where $c_{\mathbf{k}} \in \mathbb{R}$ for $|\mathbf{k}| \geq 1$. The order of correspondence of the $n$th approximant $f_{n}(\mathbf{z})$ is $v_{n}=n+1$, so that the Taylor expansion of $f_{n}(\mathbf{z})$ agrees with $L(\mathbf{z})$ up to and including the all homogeneous terms of degree $n$, that is,

$$
f_{n}(\mathbf{z})=\sum_{|\mathbf{k}|=1}^{n} c_{\mathbf{k}} \mathbf{z}^{\mathbf{k}}+\sum_{|\mathbf{k}| \geq n+1} \alpha_{\mathbf{k}}^{(n)} \mathbf{z}^{\mathbf{k}}, \quad n \geq 1,
$$

where $\alpha_{\mathbf{k}}^{(n)} \in \mathbb{R}$ for $|\mathbf{k}| \geq n+1$ and $n \geq 1$.

Proof. Since $Q_{i(k)}^{(n)}(\mathbf{0})=1$ for all $i(k) \in \mathcal{I}_{k}, 1 \leq k \leq n, n \geq 1$, then for each $i(k) \in \mathcal{I}_{k}, 1 \leq k \leq n$, $n \geq 1$, the tails $1 / Q_{i(k)}^{(n)}(\mathbf{z})$ has a formal expansion at $\mathbf{z}=\mathbf{0}$ into formal multiple power series. 
Thus, for each $n \geq 1$ the $n$th approximant $f_{n}(\mathbf{z})$ of (1) is a holomorphic function at the origin. So, let for each $n \geq 1$ the multiple power series

$$
\Lambda\left(f_{n}\right)=\sum_{|\mathbf{k}| \geq 1} \alpha_{\mathbf{k}}^{(n)} \mathbf{z}^{\mathbf{k}}
$$

where $\alpha_{\mathbf{k}}^{(n)} \in \mathbb{R},|\mathbf{k}| \geq 1, n \geq 1$, be a formal expansion of $n$th approximant $f_{n}(\mathbf{z})$ at $\mathbf{z}=\mathbf{0}$.

Now, since $Q_{i(k)}^{(n)}(\mathbf{0}) \neq 0$ for all $i(k) \in \mathcal{I}_{k}, 1 \leq k \leq n, n \geq 1$, then for each $n \geq 1$ and $m>n$ the formula (2) is valid at the origin. It follows from (2) that for any $n \geq 1$ and $m>n$ at $\mathbf{z}=\mathbf{0}$ we have

$$
\Lambda\left(f_{m}\right)-\Lambda\left(f_{n}\right)=\sum_{|\mathbf{k}| \geq n+1}\left(\alpha_{\mathbf{k}}^{(m)}-\alpha_{\mathbf{k}}^{(n)}\right) \mathbf{z}^{\mathbf{k}} .
$$

Hence for each $n \geq 1$ and $m>n$

$$
\lambda\left(\Lambda\left(f_{m}\right)-\Lambda\left(f_{n}\right)\right)=n+1
$$

tends monotonically to $+\infty$ as $n \rightarrow \infty$.

Thus, for each $n \geq 1, m>n$ and $1 \leq|\mathbf{k}| \leq n$ the equality $\alpha_{\mathbf{k}}^{(m)}=\alpha_{\mathbf{k}}^{(n)}$ is valid. Multidimensional S-fraction with independent variables (1) corresponds at $\mathbf{z}=\mathbf{0}$ to formal multiple power series (3) with $c_{\mathbf{k}}=\alpha_{\mathbf{k}}^{(|\mathbf{k}|)}$ for all $|\mathbf{k}| \geq 1$, since for $n \geq 1$

$$
L(\mathbf{z})-\Lambda\left(f_{n}\right)=\sum_{|\mathbf{k}| \geq n+1}\left(\alpha_{\mathbf{k}}^{(|\mathbf{k}|)}-\alpha_{\mathbf{k}}^{(n)}\right) \mathbf{z}^{\mathbf{k}}
$$

It follows that

$$
v_{n}=\lambda\left(L(\mathbf{z})-\Lambda\left(f_{n}\right)\right)=n+1,
$$

that is, the order of correspondence of the $n$th approximant $f_{n}(\mathbf{z})$ is $(n+1)$, and the formal multiple power series (4) is the Taylor expansion of $f_{n}(\mathbf{z})$ at $\mathbf{z}=\mathbf{0}$.

It remains to prove that this $L(\mathbf{z})$ is unique. Assume that the multidimensional $S$-fraction with independent variables (1) also corresponds to

$$
L^{*}(\mathbf{z})=\sum_{|\mathbf{k}| \geq 1} \beta_{\mathbf{k}}^{(|\mathbf{k}|)} \mathbf{z}^{\mathbf{k}}
$$

Since for any $n \geq 1$ at $\mathbf{z}=\mathbf{0}$

$$
L^{*}(\mathbf{z})-\Lambda\left(f_{n}\right)=\sum_{|\mathbf{k}| \geq n+1}\left(\beta_{\mathbf{k}}^{(|\mathbf{k}|)}-\alpha_{\mathbf{k}}^{(n)}\right) \mathbf{z}^{\mathbf{k}},
$$

then $\beta_{\mathbf{k}}^{(|\mathbf{k}|)}=\alpha_{\mathbf{k}}^{(|\mathbf{k}|)}$ for all $\mathbf{k}$ such that $1 \leq|\mathbf{k}| \leq n$ and $n \geq 1$. That is, the $L(\mathbf{z})$ is unique.

The following theorem deals with the convergence of the corresponding multidimensional $S$-fraction with independent variables to a formal multiple power series.

Theorem 2. Let the multidimensional S-fraction with independent variables (1) converges uniformly on every compact subset of the domain $D$ (which contains the origin) to the function $f(\mathbf{z})$, which is holomorphic in this domain. Then the sum of the formal multiple power series (3), which corresponds to the multidimensional S-fraction with independent variables (1), has the same value as this fraction in the domain $D$. 
Proof. We prove this by the scheme proposed in [23, Theorem 2] (see also [38, Theorem 4.1]). Let $\left\{f_{n}(\mathbf{z})\right\}$ be a sequence of approximants of (1). Since $\left\{f_{n}(\mathbf{z})\right\}$ converges uniformly on every compact subset of the domain $D$ (which contains the origin) to the function $f(\mathbf{z})$, which is holomorphic in this domain, according to Weierstrass theorem (see [37, p. 288]) for arbitrary $|\mathbf{k}| \geq 0$, we have

$$
\frac{\partial^{|\mathbf{k}|} f_{n}(\mathbf{z})}{\partial \mathbf{z}^{\mathbf{k}}} \rightarrow \frac{\partial^{|\mathbf{k}|} f(\mathbf{z})}{\partial \mathbf{z}^{\mathbf{k}}} \text { as } n \rightarrow \infty
$$

on each compact subset of the domain $D$.

And now, according to Theorem 1, the expansion of each approximant $f_{n}(\mathbf{z}), n \geq 1$, into formal multiple power series and series (3) agree for all homogeneous terms up to and including degree $n$. Then for arbitrary $\mathbf{k}$ such that $|\mathbf{k}| \geq 1$ we obtain

$$
\lim _{n \rightarrow \infty}\left(\frac{\partial^{|\mathbf{k}|} f_{n}}{\partial \mathbf{z}^{\mathbf{k}}}(\mathbf{0})\right)=\frac{\partial^{|\mathbf{k}|} f}{\partial \mathbf{z}^{\mathbf{k}}}(\mathbf{0})=\mathbf{k} ! c_{\mathbf{k}} .
$$

Hence,

$$
f(\mathbf{z})=\sum_{|\mathbf{k}| \geq 1}^{\infty} \frac{1}{\mathbf{k} !}\left(\frac{\partial^{|\mathbf{k}|} f}{\partial \mathbf{z}^{\mathbf{k}}}(\mathbf{0})\right) \mathbf{z}^{\mathbf{k}}=\sum_{|\mathbf{k}| \geq 1}^{\infty} c_{\mathbf{k}} \mathbf{z}^{\mathbf{k}}
$$

for all $\mathbf{z} \in D$.

Remark. If in Theorem 2 the convergence domain of (1) is wider than of the multiple power series (3), then the multidimensional S-fraction with independent variables (1) is an analytic continuation of $f(\mathbf{z})$ in the domain $D$.

\subsection{Algorithm}

Let $N \geq 2$. We shall construct the algorithm for the expansion of the given formal multiple power series (3) into the corresponding multidimensional $S$-fraction with independent variables (1) in much the same way as the algorithm in [19].

The following theorem is proved in [7, pp. 197-199].

Theorem 3. An S-fraction

$$
\frac{a_{1} z}{1}+\frac{a_{2} z}{1}+\frac{a_{3} z}{1}+\cdots
$$

corresponds at $z=0$ to the given formal power series

$$
L(z)=\sum_{k=1}^{\infty} \gamma_{k}(-z)^{k}
$$

where $\gamma_{k} \in \mathbb{R}$ for $k \geq 1$, if and only if the Hankel determinants associated with $L(z)$ satisfy

$$
(-1)^{n} H_{n}^{(1)}>0, \quad(-1)^{n} H_{n}^{(2)}>0 \quad \text { for all } n \geq 1 .
$$

For more details on theory of correspondence between the formal power series and the continued fraction see [35, pp. 148-160].

The process of constructing of multidimensional $S$-fraction with independent variables (1) will be shown step by step. 
Step 1. Let $\gamma_{e_{i_{1}}} \neq 0$ for $2 \leq i_{1} \leq N$. Then $L(\mathbf{z})$ can be written

$$
L(\mathbf{z})=P_{e_{0}}\left(z_{1}\right)-\sum_{i_{1}=2}^{N} \gamma_{e_{1}} z_{i_{1}} L_{e_{i_{1}}}(\mathbf{z})
$$

where

$$
P_{e_{0}}\left(z_{1}\right)=\sum_{n=1}^{\infty} \gamma_{n e_{1}}\left(-z_{1}\right)^{n}, \quad L_{e_{i_{1}}}(\mathbf{z})=\sum_{\substack{|\mathbf{k}| \geq 0 \\ k_{j}=0, i_{1}+1 \leq j \leq N}} \frac{\gamma_{\mathbf{k}+e_{i_{1}}}}{\gamma_{e_{i_{1}}}} \mathbf{z}^{\mathbf{k}}
$$

Step 2. Let $(-1)^{n} H_{e_{1}}(n)>0$ and $(-1)^{n} H_{2 e_{1}}(n)>0$ for $n \geq 1$, where

$$
H_{l e_{1}}(n)=\left|\begin{array}{cccc}
\gamma_{l e_{1}} & \gamma_{(l+1) e_{1}} & \cdots & \gamma_{(l+n-1) e_{1}} \\
\gamma_{(l+1) e_{1}} & \gamma_{(l+2) e_{1}} & \cdots & \gamma_{(l+n) e_{1}} \\
\ldots \ldots \ldots \ldots & \ldots \ldots \ldots \ldots \ldots & \ldots & \ldots \ldots \ldots \\
\gamma_{(l+n-1) e_{1}} & \gamma_{(l+n) e_{1}} & \cdots & \gamma_{(l+2 n-2) e_{1}}
\end{array}\right|, \quad l=1,2,
$$

Here $H_{e_{1}}(n)$ and $H_{2 e_{1}}(n)$ are the Hankel determinants (of dimension $n$ ) associated with the formal power series $P_{e_{0}}\left(z_{1}\right)$. Then according to Theorem 3 we have

$$
\sum_{n=1}^{\infty} \gamma_{n e_{1}}\left(-z_{1}\right)^{n} \sim \frac{a_{e_{1}} z_{1}}{1}+\frac{a_{2 e_{1}} z_{1}}{1}+\frac{a_{3 e_{1}} z_{1}}{1}+\cdots=F_{e_{0}}\left(z_{1}\right)
$$

with $a_{n e_{1}}>0$ for all $n \geq 1$. Here the symbol $\sim$ means the correspondence between $P_{e_{0}}\left(z_{1}\right)$ and $F_{e_{0}}\left(z_{1}\right)$ (at the origin). The coefficients $a_{n e_{1}}>0$ for $n \geq 1$ can be defined by the appropriate formulae [19, formulae (2.9a) and (2.9b)]. Thus we can write

$$
L(\mathbf{z}) \sim F_{e_{0}}\left(z_{1}\right)-\sum_{i_{1}=2}^{N} \gamma_{e_{i_{1}}} z_{i_{1}} L_{e_{i_{1}}}(\mathbf{z}) .
$$

Step 3. Let $(-1)^{n} H_{e_{i_{1}}}(n)>0$ and $(-1)^{n} H_{2 e_{i_{1}}}(n)>0$ for $2 \leq i_{1} \leq N$ and $n \geq 1$, where $H_{e_{i_{1}}}(n), H_{2 e_{i_{1}}}(n)$ defined by analogy to (5). Then according to Theorem 3 we have for each $2 \leq i_{1} \leq N$

$$
\sum_{n=1}^{\infty} \gamma_{n e_{i_{1}}}\left(-z_{i_{1}}\right)^{n} \sim \frac{b_{e_{i_{1}}} z_{i_{1}}}{1}+\frac{b_{e_{2 i_{1}}} z_{i_{1}}}{1}+\frac{b_{e_{3 i_{1}}} z_{i_{1}}}{1}+\cdots
$$

with $b_{n e_{i_{1}}}>0$ for all $n \geq 1$. The coefficients $b_{n e_{i_{1}}}$ for $2 \leq i_{1} \leq N$ can be defined by the appropriate formulae [19, formulae (2.9a) and (2.9b)]. Since $-\gamma_{e_{i_{1}}}=b_{e_{i_{1}}}, 2 \leq i_{1} \leq N$, we put $a_{e_{i_{1}}}=b_{e_{i_{1}}}, 2 \leq i_{1} \leq N$. Thus

$$
L(\mathbf{z}) \sim F_{e_{0}}\left(z_{1}\right)+\sum_{i_{1}=2}^{N} a_{e_{i_{1}}} z_{i_{1}} L_{e_{i_{1}}}(\mathbf{z}) .
$$

Step 4. For each $2 \leq i_{1} \leq N$, let

$$
R_{e_{i_{1}}}(\mathbf{z})=\sum_{\substack{|\mathbf{k}| \geq 0 \\ k_{j}=0, i_{1}+1 \leq j \leq N}} \gamma_{\mathbf{k}}^{e_{i_{1}}}(-\mathbf{z})^{\mathbf{k}}
$$


be reciprocal to formal multiple power series $L_{e_{i_{1}}}(\mathbf{z})$. It is known that the coefficients $\gamma_{\mathbf{k}}^{e_{i_{1}}}$, $k_{j}=0, i_{1}+1 \leq j \leq N,|\mathbf{k}| \geq 1$, of (6) are uniquely determined by a recurrent formula

$$
\gamma_{\mathbf{k}}^{e_{i_{1}}}=-\sum_{|\mathbf{r}|=1}^{|\mathbf{k}|} \gamma_{\mathbf{k}-\mathbf{r}}^{e_{i_{1}}} \frac{\gamma_{\mathbf{r}+e_{i_{1}}}}{\gamma_{e_{i_{1}}}}
$$

where $\gamma_{0}^{e_{i_{1}}}=1$, moreover, $\gamma_{\mathbf{k}}^{e_{i_{1}}}=0$, if there exists an index $j$ such that $1 \leq j \leq N$ and that $k_{j}<0$. Thus we can write

$$
L(\mathbf{z}) \sim F_{e_{0}}\left(z_{1}\right)+\sum_{i_{1}=2}^{N} \frac{a_{e_{i_{1}}} z_{i_{1}}}{R_{e_{i_{1}}}(\mathbf{z})} .
$$

The next construction of the multidimensional $S$-fraction with independent variables (1) will be carried out using the ideas laid out in Steps 1-4.

We apply Step 1 to each formal multiple power series $R_{e_{i_{1}}}(\mathbf{z})$, where $2 \leq i_{1} \leq N$. By condition $\gamma_{e_{i_{2}}}^{e_{i_{1}}} \neq 0$ for $2 \leq i_{2} \leq i_{1}$ and $2 \leq i_{1} \leq N$ we write for each $2 \leq i_{1} \leq N$

$$
R_{e_{i_{1}}}(\mathbf{z})=1+P_{e_{i_{1}}}\left(z_{1}\right)-\sum_{i_{2}=2}^{i_{1}} \gamma_{e_{i_{2}}}^{e_{i_{1}}} z_{i_{2}} L_{e_{i(2)}}(\mathbf{z})
$$

where

$$
P_{e_{i_{1}}}\left(z_{1}\right)=\sum_{n=1}^{\infty} \gamma_{n e_{1}}^{e_{i_{1}}}\left(-z_{1}\right)^{n}, \quad L_{e_{i(2)}}(\mathbf{z})=\sum_{\substack{|\mathbf{k}| \geq 0 \\ k_{j}=0, i_{2}+1 \leq j \leq N}} \frac{\gamma_{\mathbf{k}+e_{i_{2}}}^{e_{i_{1}}}}{\gamma_{e_{i_{2}}}^{e_{i_{1}}}}(-\mathbf{z})^{\mathbf{k}}
$$

Thus

$$
L(\mathbf{z}) \sim F_{e_{0}}\left(z_{1}\right)+\sum_{i_{1}=2}^{N} \frac{a_{e_{i_{1}}} z_{i_{1}}}{1+P_{e_{i_{1}}}\left(z_{1}\right)}-\sum_{i_{2}=2}^{i_{1}} \gamma_{e_{i_{2}}}^{e_{i_{1}}} z_{i_{2}} L_{e_{i(2)}}(\mathbf{z}) .
$$

Now apply Step 2 to each formal power series $P_{e_{i_{1}}}\left(z_{1}\right)$, where $2 \leq i_{1} \leq N$. Let $H_{e_{1}}^{e_{i_{1}}}(n)>0$ and $H_{2 e_{1}}^{e_{i_{1}}}(n)>0$ for $2 \leq i_{1} \leq N$ and $n \geq 1$, where

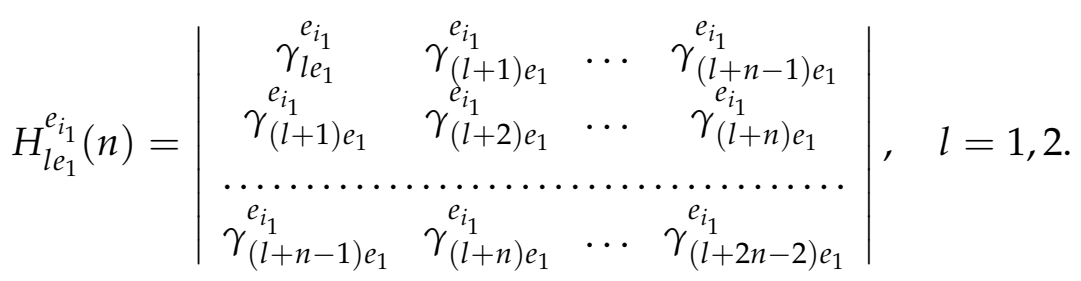

Then according to Theorem 3 we have for each $2 \leq i_{1} \leq N$

$$
\sum_{n=1}^{\infty} c_{n e_{1}}^{e_{i_{1}}} z_{1}^{n} \sim \frac{a_{e_{i_{1}}+e_{1}} z_{1}}{1}+\frac{a_{e_{i_{1}}+2 e_{1}} z_{1}}{1}+\frac{a_{e_{i_{1}}+3 e_{1}} z_{1}}{1}+\cdots=F_{e_{i_{1}}}\left(z_{1}\right)
$$

with $a_{e_{i_{1}}+n e_{1}}>0$ for all $n \geq 1$. The coefficients $a_{e_{i_{1}}+n e_{1}}$ for $n \geq 1$ can be defined by the appropriate formulae $[19$, formulae (2.10a) and (2.10b)]. Thus we can write

$$
L(\mathbf{z}) \sim F_{e_{0}}\left(z_{1}\right)+\sum_{i_{1}=2}^{N} \frac{a_{e_{i_{1}}} z_{i_{1}}}{1+F_{e_{i_{1}}}\left(z_{1}\right)}-\sum_{i_{2}=2}^{i_{1}} \gamma_{e_{i_{2}}}^{e_{i_{1}}} z_{i_{1}} L_{e_{i(2)}}(\mathbf{z}) .
$$


Next, we apply Step 3. Let $(-1)^{n} H_{e_{i_{2}}}^{e_{i_{1}}}(n)>0$ and $(-1)^{n} H_{2 e_{i_{2}}}^{e_{i_{1}}}(n)>0$ for $2 \leq i_{2} \leq i_{1}-1$, $2 \leq i_{1} \leq N$ and $n \geq 1$, where $H_{e_{i_{2}}}^{e_{i_{1}}}(n), H_{2 e_{i_{2}}}^{e_{i_{1}}}(n)$ defined by analogy to (8). Then according to Theorem 3 we have for each $2 \leq i_{2} \leq i_{1}-1$ and $2 \leq i_{1} \leq N$

$$
\sum_{n=1}^{\infty} \gamma_{n e_{i_{2}}}^{e_{i_{1}}}\left(-z_{i_{2}}\right)^{n} \sim \frac{b_{e_{i_{1}}+e_{i_{2}}} z_{i_{2}}}{1}+\frac{b_{e_{i_{1}}+2 e_{i_{2}}} z_{i_{2}}}{1}+\frac{b_{e_{i_{1}}+3 e_{i_{2}} z_{i_{2}}}}{1}+\ldots
$$

with $b_{e_{1}+n e_{i_{2}}}>0$ for all $n \geq 1$. The coefficients $b_{e_{i_{1}}+n e_{i_{2}}}$ for $2 \leq i_{1} \leq N$ can be defined by the appropriate formulae [19, formulae (2.10a) and (2.10b)]. Since for $2 \leq i_{2} \leq i_{1}-1$ and $2 \leq i_{1} \leq N$

$$
-\gamma_{e_{i_{2}}}^{e_{i_{1}}}=c_{e_{e_{2}}}^{e_{i_{1}}}=-\frac{c_{e_{i(2)}}}{c_{e_{i_{1}}}}=b_{\left.e_{i(2)}\right)^{\prime}} \quad-\gamma_{e_{i_{1}}}^{e_{i_{1}}}=c_{e_{i_{1}}}^{e_{i_{1}}}=-\frac{c_{2 e_{i_{1}}}}{c_{e_{i_{1}}}}=b_{2 e_{i_{1}}},
$$

then we put $a_{e_{i(2)}}=b_{e_{i(2)}}, a_{2 e_{i_{1}}}=b_{2 e_{i_{1}}}$ for all $2 \leq i_{2} \leq i_{1}-1$ and $2 \leq i_{1} \leq N$. Thus

$$
L(\mathbf{z}) \sim F_{e_{0}}\left(z_{1}\right)+\sum_{i_{1}=2}^{N} \frac{a_{e_{i_{1}}} z_{i_{1}}}{1+F_{e_{i_{1}}}\left(z_{1}\right)}+\sum_{i_{2}=2}^{i_{1}} a_{e_{i(2)}} z_{i_{2}} L_{e_{i(2)}}(\mathbf{z}) .
$$

At last, applying Step 4 to each formal multiple power series $L_{e_{i(2)}}(\mathbf{z})$, where $2 \leq i_{2} \leq i_{1}$ and $2 \leq i_{1} \leq N$, we can write

$$
L(\mathbf{z}) \sim F_{e_{0}}\left(z_{1}\right)+\sum_{i_{1}=2}^{N} \frac{a_{e_{i_{1}}} z_{i_{1}}}{1+F_{e_{i_{1}}}\left(z_{1}\right)}+\sum_{i_{2}=2}^{i_{1}} \frac{a_{e_{i(2)}} z_{i_{2}}}{R_{e_{i(2)}}(\mathbf{z})},
$$

where $R_{e_{i(2)}}(\mathbf{z})$ is reciprocal to formal multiple power series $L_{e_{i(2)}}(\mathbf{z})$.

Further construction of the multidimensional $S$-fraction with independent variables (1) is the sequential application of Steps 1-4 to all formal power series that are in the denominators of the ending partial quotients of the finite branches of the branched continued fraction with independent variables. As a result, computing the coefficients

$$
\gamma_{\mathbf{k}}^{e_{i_{1}}}, \quad|\mathbf{k}| \geq 1, k_{j}=0, i_{1}+1 \leq j \leq N, 2 \leq i_{1} \leq N,
$$

by the recurrent formula (7) and the coefficients

$$
\gamma_{\mathbf{k}}^{e_{i(k)}}, \quad|\mathbf{k}| \geq 1, k_{j}=0, i_{k}+1 \leq j \leq N, k \geq 2,2 \leq i_{p} \leq i_{p-1}, 1 \leq p \leq k,
$$

by the recurrent formula

$$
\gamma_{\mathbf{k}}^{e_{i(k)}}=-\sum_{|\mathbf{r}|=1}^{|\mathbf{k}|} \gamma_{\mathbf{k}-\mathbf{r}}^{e_{i(k)}} \frac{\gamma_{\mathbf{r}+e_{i_{k}}}^{e_{i(k-1)}}}{\gamma_{e_{i_{k}}}^{e_{i(k-1)}}}
$$

where $\gamma_{\mathbf{0}}^{e_{i(k)}}=1$, moreover, $\gamma_{\mathbf{k}}^{e_{i(k)}}=0$, if there exists an index $j$ such that $1 \leq j \leq N$ and that $k_{j}<0$, provided that for $1 \leq i_{1} \leq N$ and $n \geq 1$

$$
(-1)^{n} H_{e_{i_{1}}}(n)>0, \quad(-1)^{n} H_{2 e_{i_{1}}}(n)>0,
$$

where

$$
H_{l e_{i_{1}}}(n)=\left|\begin{array}{cccc}
\gamma_{l e_{i_{1}}} & \gamma_{(l+1) e_{i_{1}}} & \ldots & \gamma_{(l+n-1) e_{i_{1}}} \\
\gamma_{(l+1) e_{i_{1}}} & \gamma_{(l+2) e_{i_{1}}} & \ldots & \gamma_{(l+n) e_{i_{1}}} \\
\ldots \ldots \ldots \ldots \ldots & \ldots \ldots \ldots \ldots \ldots \ldots \ldots \ldots \\
\gamma_{(l+n-1) e_{i_{1}}} & \gamma_{(l+n) e_{i_{1}}} & \ldots & \gamma_{(l+2 n-2) e_{i_{1}}}
\end{array}\right|, \quad l=1,2
$$


and for $1 \leq i_{k+1} \leq i_{k}-1,2 \leq i_{p} \leq i_{p-1}, 1 \leq p \leq k, k \geq 1$ and $n \geq 1$

$$
(-1)^{n} H_{e_{i_{k+1}}}^{e_{i(k)}}(n)>0, \quad(-1)^{n} H_{2 e_{i_{k+1}}}^{e_{i(k)}}(n)>0,
$$

where

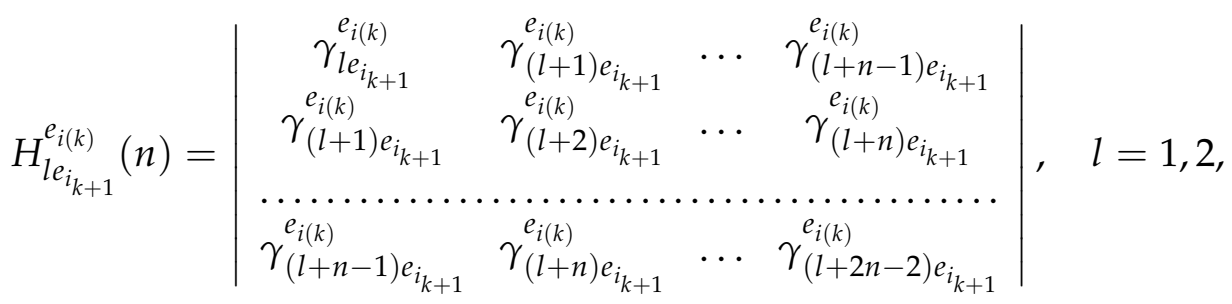

for formal multiple power series (3) we obtain multidimensional $S$-fraction with independent variables (1), where the $a_{e_{i(k)}}$ for all $e_{i(k)} \in \mathcal{E}_{k}$ and $k \geq 1$ can be defined by the appropriate formulae [19, formulae (2.9a), (2.9b), (2.10a), and (2.10b)].

Finally, it follows immediately from [19, Theorem 2.1] that the above-mentioned multidimensional $S$-fraction with independent variables (1) corresponds to the formal multiple power series (3) at $\mathbf{z}=\mathbf{0}$.

Hence, we have the following result.

Theorem 4. A multidimensional S-fraction with independent variables (1) corresponds at $\mathbf{z}=\mathbf{0}$ to the given formal multiple power series (3) if and only if the inequalities (9) for all $1 \leq i_{1} \leq N, n \geq 1$, and the inequalities (10) for all $1 \leq i_{k+1} \leq i_{k}-1,2 \leq i_{p} \leq i_{p-1}, 1 \leq p \leq k$, $k \geq 1, n \geq 1$, are satisfied.

\subsection{Convergence}

Convergence criteria have been given in $[2,3,5,6,9,12,20]$ for the multidimensional $S$-fractions with independent variables. However, the problem of the widest convergence domains and estimates of convergence rate for the above-mentioned branched continued fractions remains open.

\section{Some branched continued fraction expansions}

\subsection{Functions of several variables}

For some functions of several variables we give their multidimensional $S$-fractions with independent variables representations, each with their domain of convergence in the complex space. The speed of convergence of some given multidimensional $S$-fractions with independent variables are illustrated with some plots and typical evaluations.

Example 1. The function of two variables

$$
f\left(z_{1}, z_{2}\right)=\sqrt{z_{2}} \arctan \sqrt{\frac{z_{2}}{1+z_{1}}}
$$

has a formal double power series given by

$$
L\left(z_{1}, z_{2}\right)=\left(\sum_{l=0}^{\infty} \frac{(-1 / 2)_{l}}{l !}\left(-z_{2}\right)^{l}\right) \sum_{k=1}^{\infty} \frac{(-1)^{k-1}}{2 k-1}\left(z_{2} \sum_{l=0}^{\infty}\left(-z_{1}\right)^{l}\right)^{k},
$$


where $(\cdot)_{k}$ is the Pochhammer symbol defined for any complex number $\alpha$ and non-negative integers $k$ by $(\alpha)_{0}=1$ and $(\alpha)_{k}=\alpha(\alpha+1) \ldots(\alpha+k-1)$. Applying the above-mentioned algorithm to (12) yields the two-dimensional $S$-fraction with independent variables

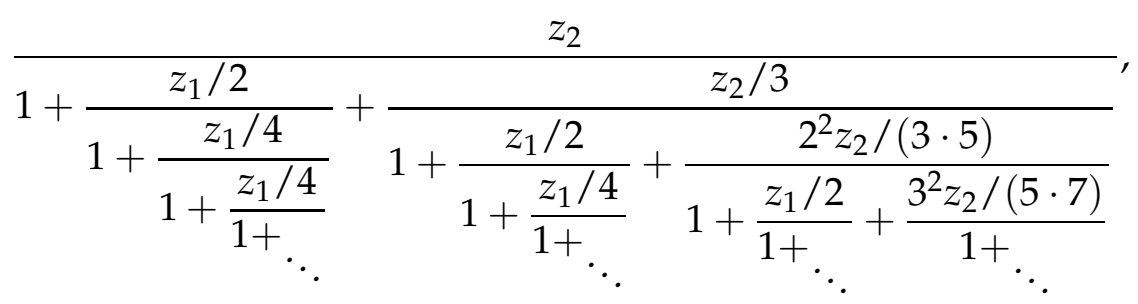

where $\left|\arg \left(z_{1}+1 / 2\right)\right|<\pi,\left|\arg \left(z_{2}\right)\right|<\pi$.

Plots of the values of the $n$th approximants of the two-dimensional S-fraction with independent variables (13) are shown in Figure 1. Here we can see the so-called "fork property" for a branched continued fraction with positive elements (see [1, Remark 4] or [8, p. 29]). That is, the plots of the values of even (odd) approximations of (13) approaches from below (above) to the plot of the function of two variables (11). Plots of the absolute error of the $n$th approx-

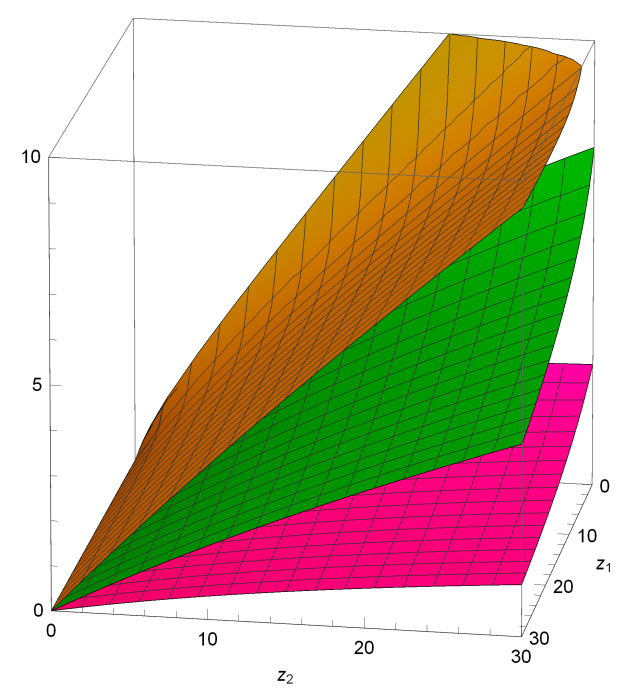

(a) $\square-2 \mathrm{nd}, \square-(11), \square-3 \mathrm{rd}$

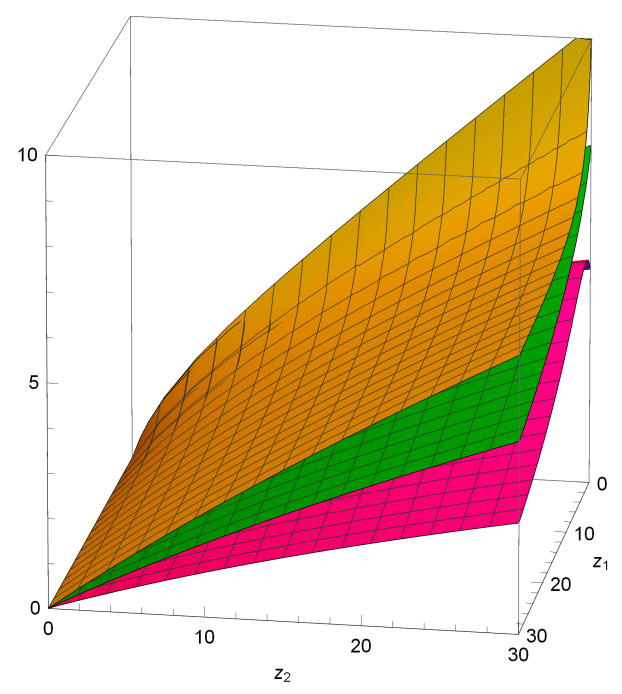

(b) $\square-4$ th, $\square-(11), \square-5$ th

Figure 1. The plots of values of the $n$th approximants of (13)

imants of (13) are shown in Figure 2. In these plots, we can see that the rate of convergence of the two-dimensional S-fraction with independent variables (13), despite its structure, in the direction of growth of the variable $z_{2}$ is better than $z_{1}$. This is explained by the fact that in (13) branches with variable $z_{1}$ are periodic continued fractions, which are known to have a slow rate of convergence compared to others (see [35, Chapter 8]).

Finally, the numerical illustration of (12) and (13) is given in the Table 1. Here we compare the relative errors of the approximation of function of two variables (11) by the partial sums of the formal double power series and the approximants of the corresponding two-dimensional $S$-fraction with independent variables. As a results, the $n$th approximant of (13) is eventually a better approximation to (11) than the $n$th partial sum of (12) is. 


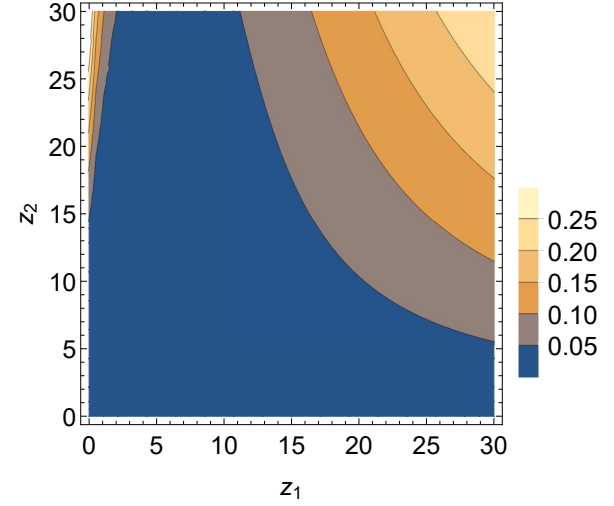

(a) 10 th

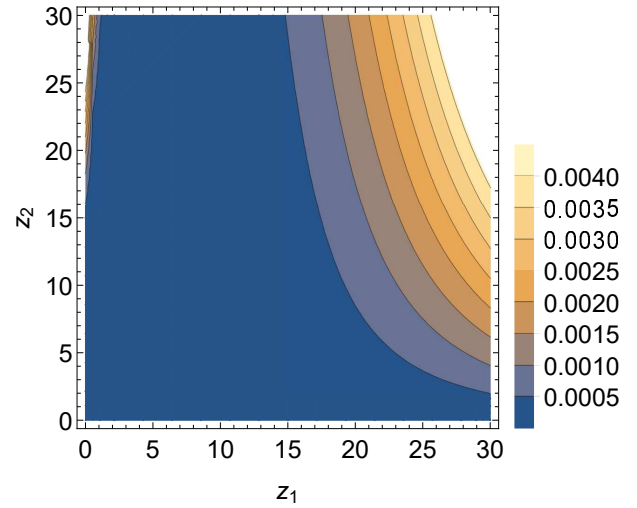

(b) 20 th

Figure 2. The plots of absolute error of the $n$th approximants of (13)

\begin{tabular}{lccc}
\hline$\left(z_{1}, z_{2}\right)$ & $(11)$ & $(12)$ & $(13)$ \\
\hline$(-0.9,0.9)$ & 1.184948863 & $4.22581 \times 10^{-2}$ & $1.26921 \times 10^{-2}$ \\
$(-0.1,0.8)$ & 0.676159596 & $1.05461 \times 10^{-2}$ & $1.11297 \times 10^{-4}$ \\
$(0.2,0.7)$ & 0.545712469 & $4.26445 \times 10^{-2}$ & $6.72182 \times 10^{-5}$ \\
$(0.8,0.9)$ & 0.58389532 & $5.2986 \times 10^{-1}$ & $4.16608 \times 10^{-4}$ \\
$(0.9,0.1)$ & 0.071313601 & $1.35009 \times 10^{-1}$ & $2.23898 \times 10^{-4}$ \\
$(0.2,0.1)$ & 0.088871039 & $3.3224 \times 10^{-4}$ & $7.83672 \times 10^{-7}$ \\
$(-0.1,0.1)$ & 0.101746459 & $2.38433 \times 10^{-7}$ & $8.88188 \times 10^{-9}$ \\
$(-0.8,0.2)$ & 0.351240737 & $6.24974 \times 10^{-2}$ & $7.80438 \times 10^{-3}$ \\
\hline
\end{tabular}

Table 1. Relative error of 5th partial sum and 5th approximant

Example 2. For

$$
f\left(z_{1}, z_{2}\right)=\sqrt{1+z_{1}} \ln \left(1+\frac{z_{2}}{1+z_{1}}\right)
$$

we have

$$
L\left(z_{1}, z_{2}\right)=\left(\sum_{l=0}^{\infty} \frac{(-1 / 2)_{l}}{l !}\left(-z_{2}\right)^{l}\right) \sum_{k=1}^{\infty} \frac{(-1)^{k-1}}{k}\left(z_{2} \sum_{l=0}^{\infty}\left(-z_{1}\right)^{l}\right)^{k}
$$

and find that $a_{1, l}=1 / 2, a_{k, l}=1 / 4, a_{0,1}=1, a_{0,2 l}=1 /(4 l-2)$, and $a_{0,2 l+1}=1 /(4 l+2)$ for $k \geq 2$ and $l \geq 1$. The two-dimensional $S$-fraction with independent variables representation of the function of two variables (14) is

$$
\frac{a_{0,1} z_{2}}{1+\frac{a_{1,1} z_{1}}{1+\frac{a_{2,1} z_{1}}{1+}}+\frac{a_{0,2} z_{2}}{1+\frac{a_{1,2} z_{1}}{1+}+\frac{a_{0,3} z_{2}}{1+}}},
$$

where $\left|\arg \left(z_{k}+l_{k} / 4\right)\right|<\pi, l_{k}>0, l_{1}+l_{2} \leq 2$ for $k=1,2$.

Plots of the values and absolute error of the $n$th approximants of the two-dimensional $S$-fraction with independent variables (16) are shown in Figures 3 and 4, respectively. A numerical illustration of (15) and (16) is given in the Table 2. Here we have results like to the results in Example 1.

Note that the branched continued fraction expansion of the function of several variables given in [19, Example 3.2] is also multidimensional $S$-fraction with independent variables. 


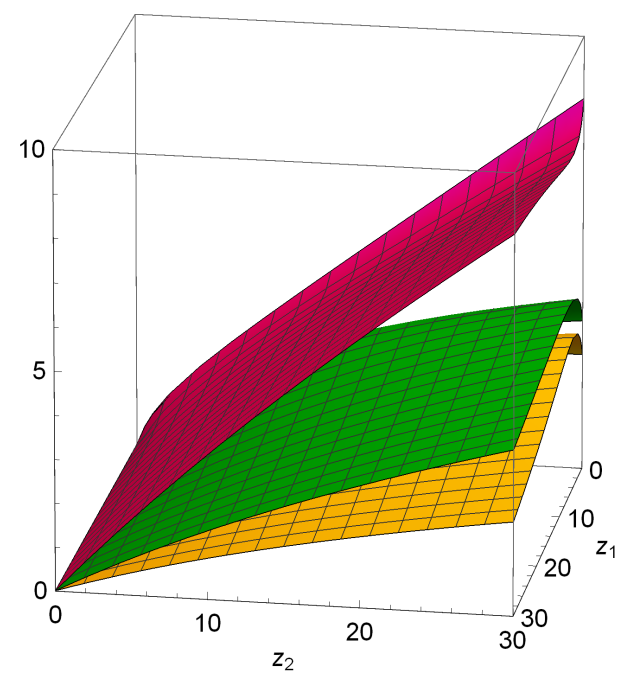

(a) $\square-3 \mathrm{rd}, \square-(14), \square-4$ th

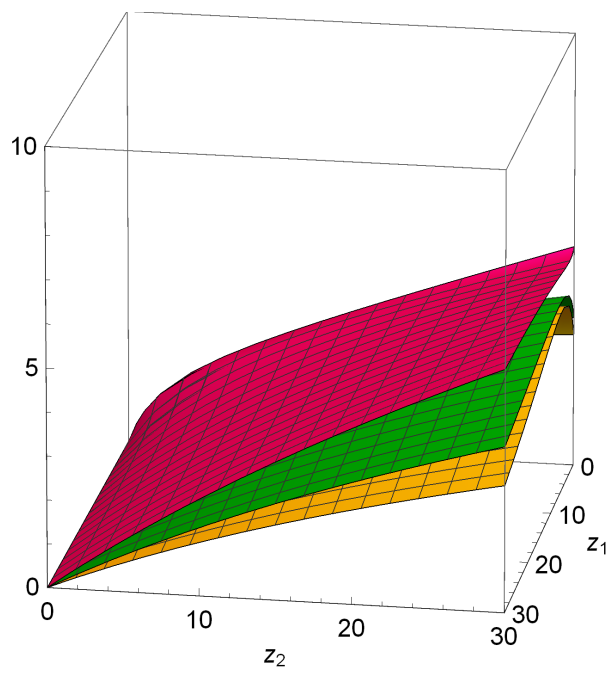

(b) $\square-5$ th, $\square-(14), \square-6$ th

Figure 3. The plots of values of the $n$th approximants of (16)

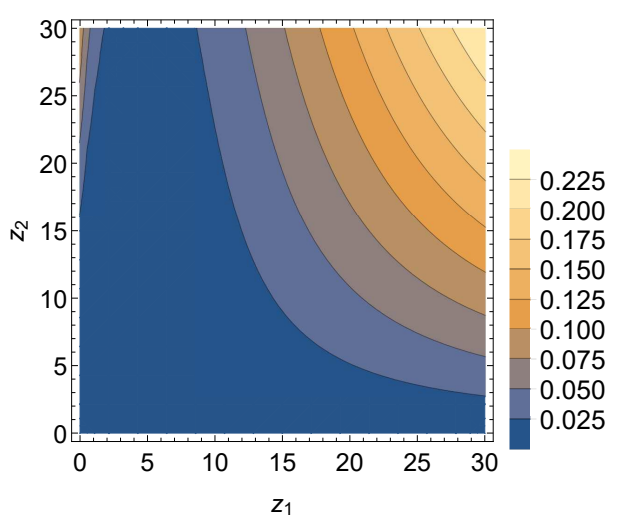

(a) $10 \mathrm{th}$

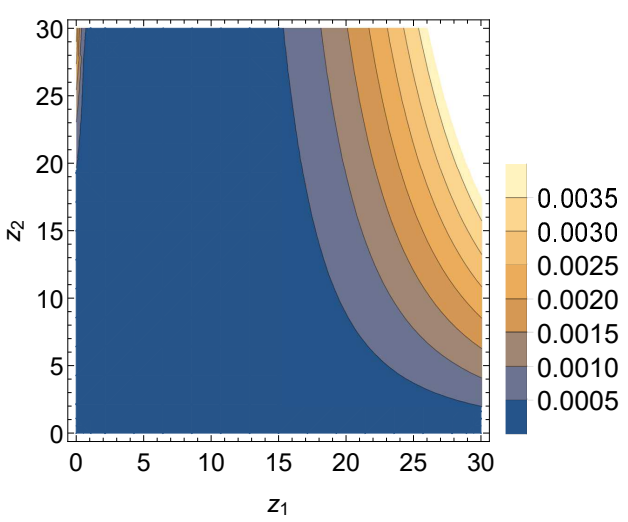

(b) 20 th

Figure 4. The plots of absolute error of the $n$th approximants of (16)

\begin{tabular}{lccc}
\hline$\left(z_{1}, z_{2}\right)$ & $(14)$ & $(15)$ & $(16)$ \\
\hline$(-0.9,0.9)$ & 0.72814134 & $4.55047 \times 10^{-2}$ & $3.53063 \times 10^{-3}$ \\
$(-0.1,1)$ & 0.708869823 & $2.39618 \times 10^{-2}$ & $4.93611 \times 10^{-8}$ \\
$(0.1,0.1)$ & 0.091258302 & $1.15296 \times 10^{-8}$ & $3.04143 \times 10^{-16}$ \\
$(0.2,-0.9)$ & -1.518609386 & $3.86895 \times 10^{-3}$ & $1.8452 \times 10^{-5}$ \\
$(1.1,-0.8)$ & -0.694967418 & $3.35801 \times 10^{-2}$ & $5.33114 \times 10^{-11}$ \\
$(2,0.1)$ & 0.056793639 & $1.00592 \times 10^{0}$ & $3.98912 \times 10^{-6}$ \\
$(-0.1,5)$ & 1.783821412 & $1.0 \times 10^{0}$ & $3.20199 \times 10^{-4}$ \\
$(10,10)$ & 2.144619685 & $1.0 \times 10^{0}$ & $5.26825 \times 10^{-3}$ \\
$(1,40)$ & 4.305604922 & $1.0 \times 10^{0}$ & $1.9013 \times 10^{-2}$ \\
$(50,2)$ & 0.274704191 & $1.0 \times 10^{0}$ & $1.27757 \times 10^{-1}$ \\
\hline
\end{tabular}

Table 2. Relative error of 10th partial sum and 10th approximants 


\subsection{Mathematical constants}

The calculation of mathematical constants has been a topic of investigation for mathematicians throughout the centuries [15, Chapter 10]. Some modern applied problems of approximation of numbers by continued fraction expansions are considered in [27, 28, 31,36]. Here we show how branched continued fractions can be relevant in connection with some of the important mathematical constants.

Example 3. An important irrational number $\sqrt{2}$ is the diagonal of a unit square, sometimes called Pythagoras' constant. The numerical value of this algebraic number is

$$
\sqrt{2}=1.414213562373 \ldots
$$

The constant $\sqrt{2}$ can be obtained from [19, Example 3.4]

$$
\begin{aligned}
& \sqrt{2}=1+\frac{1 / 6}{1+\frac{/ 12}{1+\frac{1 / 12}{1+}}}+\frac{1 / 6}{1+\frac{1 / 6}{1+\frac{1 / 12}{1+} \vartheta_{\ddots}}+\frac{1 / 12}{1+\frac{1 / 6}{1+}+\frac{1 / 12}{1+} \cdot}} \\
& +\frac{1 / 6}{1+\frac{1 / 6}{1+\frac{1 / 12}{1+}}+\frac{1 / 6}{1+\frac{1 / 6}{1+}+\frac{1 / 12}{1+}}+\frac{1 / 12}{1+\frac{1 / 6}{1+}+\frac{1 / 6}{{ }_{\ddots}}+\frac{1 / 12}{1+}++_{\ddots}}} .
\end{aligned}
$$

The approximants $f_{4}$ and $f_{5}$ yield

$$
1.414130953<f_{4}=\frac{1101279537351}{778767719747}<\sqrt{2}<\frac{2566545667666280407}{1814812946556935598}=f_{5}<1.414220497,
$$

where

$$
\left|f_{4}-f_{5}\right| \leq 8.95447 \times 10^{-5}
$$

Example 4. The one of the most important mathematical constant $\pi$ is defined as the area enclosed by the unit circle. The numerical value of this algebraic number is

$$
\pi=3.141592653589793238 \ldots
$$

The expression

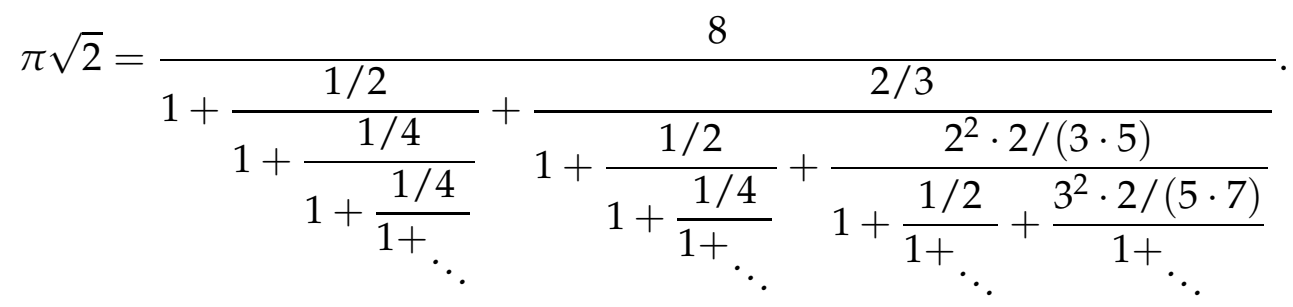

follows immediately from the Example 1. The value $\pi \sqrt{2}$ is enclosed by

$$
4.441178421<f_{6}=\frac{199851135120}{44999573573}<\pi \sqrt{2}<\frac{750454176962984}{168898725446623}=f_{7}<4.443219894
$$

with

$$
\left|f_{6}-f_{7}\right| \leq 2.041473 \times 10^{-3}
$$


Thus, several numerical experiments show the efficiency, power and feasibility of using the branched continued fractions in order to numerically approximate certain functions of several variables from their formal multiple power series.

\section{References}

[1] Antonova T., Dmytryshyn R., Kravtsiv V. Branched continued fraction expansions of Horn's hypergeometric function $\mathrm{H}_{3}$ ratios. Math. 2021, 9 (2), 148. doi:10.3390/math9020148

[2] Antonova T.M., Dmytryshyn R.I. Truncation error bounds for branched continued fraction $\sum_{i_{1}=1}^{N} \frac{a_{i(1)}}{1}+\sum_{i_{2}=1}^{i_{1}} \frac{a_{i(2)}}{1}+\sum_{i_{3}=1}^{i_{2}} \frac{a_{i(3)}}{1}+\ldots$ Ukrainian Math. J. 2020, 72 (7), 1018-1029. doi:10.1007/s11253020-01841-7 (translation of Ukrain. Math. Zh. 2020, 72 (7), 877-885. doi:10.37863/umzh.v72i7.2342 (in Ukrainian))

[3] Antonova T.M., Dmytryshyn R.I. Truncation error bounds for branched continued fraction whose partial denominators are equal to unity. Mat. Stud. 2020, 54 (1), 3-14. doi:10.30970/ms.54.1.3-14

[4] Antonova T.M., Hoyenko N.P. Approximation of Lauricella's functions $F_{D}$ ratio by Nörlund's branched continued fraction in the complex domain. Mat. Metody Fiz.-Mekh. Polya 2004, 47 (2), 7-15. (in Ukrainian)

[5] Baran O.E. An analog of the Vorpits'kii convergence criterion for branched continued fractions of special form. J. Math. Sci. (N.Y.) 1998, 90 (5), 2348-2351. doi:10.1007/BF02433964 (translation of Mat. Metody Fiz.-Mekh. Polya 1996, 39 (2), 35-38. (in Ukrainian))

[6] Baran O.E. Twin circular domains of convergence of branched continued fractions with inequivalent variables. J. Math. Sci. (N.Y.) 2011, 174 (2), 209-218. doi:10.1007/s10958-011-0291-0 (translation of Mat. Metody Fiz.-Mekh. Polya 2009, 52 (4), 73-80. (in Ukrainian))

[7] Baker G.A., Graves-Morris P. Padé approximants. Cambridge Univ. Press, Cambridge, 1996. doi:10.1017/CBO9780511530074

[8] Bodnar D.I. Branched continued fractions. Naukova Dumka, Kyiv, 1986. (in Russian)

[9] Bodnar D.I., Bilanyk I.B. Estimates of the rate of pointwise and uniform convergence for branched continued fractions with nonequivalent variables. Mat. Metody Fiz.-Mekh. Polya 2019, 62 (4), 72-82. (in Ukrainian)

[10] Bodnar D.I., Dmytryshyn R.I. Multidimensional associated fractions with independent variables and multiple power series. Ukrainian Math. J. 2019, 71 (3), 370-386. doi:10.1007/s11253-019-01652-5 (translation of Ukrain. Mat. Zh. 2019, 71 (3), 325-339. (in Ukrainian))

[11] Bodnar D.I. Investigation of the convergence of one class of branched continued fractions. In: Scorobogatko V.Ya. (Ed.) Continued fractions and their applications. Inst. Math. AN USSR, Kyiv, 1976. (In Russian)

[12] Bodnar O.S., Dmytryshyn R.I., Sharyn S.V. On the convergence of multidimensional S-fractions with independent variables. Carpathian Math. Publ. 2020, 12 (2), 353-359. doi:10.15330/cmp.12.2.353-359

[13] Cuyt A. A review of multivariate Padé approximation theory. J. Comput. Appl. Math. 1985, 12-13, 221-232. doi:10.1016/0377-0427(85)90019-6

[14] Cuyt A., Verdonk B. A review of branched continued fraction theory for the construction of multivariate rational approximants. Appl. Numer. Math. 1988, 4 (2-4), 263-271. doi:10.1016/0168-9274(83)90006-5

[15] Cuyt A., Petersen V.B., Verdonk B., Waadeland H., Jones W.B. Handbook of continued fractions for special functions. Springer, Dordrecht, 2008.

[16] Dmytryshyn R.I. Associated branched continued fractions with two independent variables. Ukrainian Math. J. 2015, 66 (9), 1312-1323. doi:10.1007/s11253-015-1011-6 (translation of Ukrain. Mat. Zh. 2014, 66 (9), 1175-1184. (in Ukrainian))

[17] Dmytryshyn R.I. Convergence of multidimensional A- and J-fractions with independent variables. Comput. Methods Funct. Theory 2021. doi:10.1007/s40315-021-00377-6 
[18] Dmytryshyn R.I. Convergence of some branched continued fractions with independent variables. Mat. Stud. 2017, 47 (2), 150-159. doi:10.15330/ms.47.2.150-159

[19] Dmytryshyn R.I. Multidimensional regular C-fraction with independent variables corresponding to formal multiple power series. Proc. Roy. Soc. Edinburgh Sect. A 2020, 150 (4), 153-1870. doi:10.1017/prm.2019.2

[20] Dmytryshyn R.I. On some of convergence domains of multidimensional S-fractions with independent variables. Carpathian Math. Publ. 2019, 11 (1), 54-58. doi:10.15330/cmp.11.1.54-58

[21] Dmytryshyn R.I. On the expansion of some functions in a two-dimensional g-fraction with independent variables. J. Math. Sci. 2012, 181 (3), 320-327. doi:10.1007/s10958-012-0687-5 (translation of Mat. Metody Fiz.-Mekh. Polya 2010, 53 (4), 56-69. (in Ukrainian))

[22] Dmytryshyn R.I. The multidimensional generalization of g-fractions and their application. J. Comp. and Appl. Math. 2004, 164-165, 265-284. doi:10.1016/S0377-0427(03)00642-3

[23] Dmytryshyn R.I. The two-dimensional g-fraction with independent variables for double power series. J. Approx. Theory 2012, 164 (12), 1520-1539. doi:10.1016/j.jat.2012.09.002

[24] Dmytryshyn R.I. Two-dimensional generalization of the Rutishauser qd-algorithm. J. Math. Sci. 2015,208 (3), 301-309. doi:10.1007/s11253-015-1011-6 (translation of Mat. Metody Fiz.-Mekh. Polya 2014, 56 (4), 6-11. (in Ukrainian))

[25] Holub A.P., Pozharskiy O.A., Chernetska L.O. Generalized moment representations and multivariate multipoint Padé approximants. Ukrainian Math. J. 2020, 71 (10), 1522-1540. doi:10.1007/s11253-020-01729-6 (translation of Ukrain. Mat. Zh. 2019, 71 (10), 1331-1346. (in Ukrainian))

[26] Hoyenko N., Antonova T., Rakintsev S. Approximation for ratios of Lauricella-Saran fuctions $F_{S}$ with real parameters by a branched continued fractions. Math. Bul. Shevchenko Sci. Soc. 2011, 8, 28-42. (in Ukrainian)

[27] Lascu D., Sebe G.I. A Gauss-Kuzmin-Lévy theorem for Rényi-type continued fractions. Acta Arith. 2020,193 (3), 283-292.

[28] Lascu D., Sebe G.I. A Lochs-type approach via entropy in comparing the efficiency of different continued fraction algorithms. Math. 2021, 9 (3), 255. doi:10.3390/math9030255

[29] Murphy J.A., O'Donohoe M.R. A two-variable generalization of the Stieltjes-type continued fraction. J. Comput. Appl. Math. 1978, 4 (3), 181-190. doi:10.1016/0771-050x(78)90002-5

[30] Komatsu T. Branched continued fractions associated with Hosoya index of the tree graph. MATCH Commun. Math. Comput. Chem. 2020, 84 (2), 399-428.

[31] Komatsu T. Continued fraction expansions of the generating functions of Bernoulli and related numbers. Indag. Math. 2020, 31 (4), 695-713. doi:10.1016/j.indag.2020.06.006

[32] Kuchminska K.Y., Vozna S.M. Development of an N-multiple power series into an N-dimensional regular C-fraction. J. Math. Sci. 2020, 246 (2), 201-208. doi:10.1007/s10958-020-04730-3 (translation of Mat. Metody Fiz.-Mekh. Polya 2017, 60 (3), 70-75. (in Ukrainian))

[33] Kuchminskaya K., Siemaszko W. Rational approximation and interpolation of functions by branched continued fractions. In: Gilewicz J., Pindor M., Siemaszko W. (Eds.) Rational approximation and its applications in mathematics and physics. Lecture Notes in Mathematics, 1237. Springer, Berlin, Heidelberg, 1987.

[34] Pétréolle M., Sokal A.D., Zhu B.-X. Lattice paths and branched continued fractions: An infinite sequence of generalizations of the Stieltjes-Rogers and Thron-Rogers polynomials, with coefficientwise Hankel-total positivity. arXiv 2020, arXiv:1807.03271v2.

[35] Jones W.B., Thron W.J. Continued fractions: analytic theory and applications. Addison-Wesley Pub. Co., Reading, MA, 1980. 
[36] Sebe G.I., Lascu D. Convergence rate for Rényi-type continued fraction expansions. Period. Math. Hung. 2020, 81 (2), 239-249. doi:10.1007/s10998-020-00325-2

[37] Shabat B.V. Introduce in the complex analysis. Nauka, Moscow, 1969. (in Russian)

[38] Siemaszko W. Branched continued fractions for double power series. J. Comput. Appl. Math. 1980, 6 (2), 121-125. doi:10.1016/0771-050x(80)90005-4

[39] Scorobogatko V.Ya. Theory of branched continued fractions and its applications in computational mathematics. Nauka, Moscow, 1983. (in Russian)

Received 18.04.2021

Амитришин Р.І., Шарин С.В. Наближення функиій багатьох змінних багатовимірними S-дробами з нерівнозначними змінними // Карпатські матем. публ. - 2021. - Т.13, №3. - С. 592-607.

У статті розглядається задача наближення функцій багатьох змінних гіллястими ланцюговими дробами, зокрема, вивчається відповідність між формальним кратним степеневим рядом і так званим “багатовимірним $S$-дробом з нерівнозначними змінними”. Встановлено необхідні та достатні умови для розвинення формального кратного степеневого ряду у віАповіАний багатовимірний $S$-дріб з нерівнозначними змінними і прикладами показано ефективність та доцільність використання гіллястих ланщюгових дробів для чисельного наближення деяких функцій багатьох змінних на відміну від їх формальних кратних степеневих рядів.

Ключові слова і фрази: гіллястий ланщюговий дріб, неперервний дріб, кратний степеневий ряд, алгоритм, відповідність. 